\title{
Laboratory Activities to Illustrate the Importance of Low Cycle Fatigue
}

Dr. Stephen Boedo, Rochester Institute of Technology (COE)

Dr. Elizabeth A. DeBartolo, Rochester Institute of Technology (COE)

Elizabeth A. DeBartolo is an Associate Professor in the Mechanical Engineering Department at the Rochester Institute of Technology. She earned her B.S.E. at Duke University in 1994 and her Ph.D. at Purdue University in 2000. She works with students on assistive device design and determining mechanical properties of materials. DeBartolo serves on her college's leadership teams for both multi-disciplinary capstone design and outreach program development.

Matthew Kasemer, Rochester Institute of Technology 


\section{Laboratory and Classroom Study of Low Cycle Fatigue}

\section{Introduction}

Low cycle fatigue theory (LCF) and linear elastic fracture mechanics [1,2] are important topics for mechanical engineering students to learn and understand. Essential in broadening the scope and depth of students' knowledge of mechanics, these topics create a better-versed engineer with experience in topics necessary in many industries. Current required coursework in Mechanical Engineering at RIT is insufficient in that it only covers HCF theory and bypasses fracture mechanics altogether. An important gap in student understanding of these failure theories is that they tend to use the techniques taught in class in any similar situation. HCF is taught as it relates to machine design, and is therefore focused on application to steels, yet students may try to use this tool in analyses where plastic strain is a significant contributor to failure, or where the components being analyzed are made from aluminum or other material that does not have a welldefined endurance limit. Similarly, the only static failure theories our students see are based on undamaged materials, not materials that may have pre-existing cracks. Students will leave the class and be tempted to try to apply these theories to cracked bodies that could fail at lower applied stresses.

The importance of incorporating laboratory fatigue testing in the curriculum has been recently addressed at several universities. A high-frequency fatigue tester has been developed to provide HCF results in as little as 30 minutes [3], but attention to resonance effects and critical speeds needs to be carefully addressed in such a test setup. A low cycle, finite life fatigue experimental setup was recently developed [4] using cantilever beams of variable cross-section to investigate effects of stress concentrations and material selection on LCF predictions. A methodology of incorporating numerical experiments of LCF and HCF in the classroom using a commercially available finite element software package has been recently reported [5]. A redevelopment of an existing vibrations laboratory to include fatigue life evaluation has also been reported [6,7].

This paper describes hands-on laboratory activities that illustrate the limitations of HCF prediction methods covered in detail in a third-year Design of Machine Elements (DME) class. This does not necessarily require that significant material needs to be added to the class, but only that students are made aware that what is taught to them is not universally applicable, and that they must understand the problem at hand before applying a particular fatigue model. Fatigue specimens and test protocol were designed by an undergraduate mechanical engineering student, as part of an independent study project.

\section{Background}

The current course at RIT that covers fatigue, Design of Machine Elements (DME), focuses only on stress-based high cycle theories with no mention of fracture mechanics. While high cycle fatigue theory is generally acceptable in applications for materials such as steel under low stress conditions, the theory is insufficient in describing some of the more complex phenomena witnessed under more intense loading in different materials, or crack-growth-based life prediction methods that require the use of fracture mechanics. High cycle fatigue theory focuses mainly on specimens that are subjected to relatively low stress situations where the cyclic 
deformation is entirely elastic. Cyclic loading in the plastic region results in low cycle count to failure. Low cycle fatigue is a strain based theory that better represents the behavior of materials subjected to cyclic loading resulting in plastic deformation. Low cycle fatigue theory takes into account both the elastic and plastic strain, while high cycle fatigue theory ignores plasticity. A typical strain-life plot is seen in Figure 1, with the elastic strain $\left(\Delta \varepsilon_{\mathrm{e}}\right)$, plastic strain $\left(\Delta \varepsilon_{\mathrm{p}}\right)$, and total strain $(\Delta \varepsilon)$ curves shown, along with a line marking the transition life, where the fatigue failure mode changes from elastic-dominant to plastic-dominant.

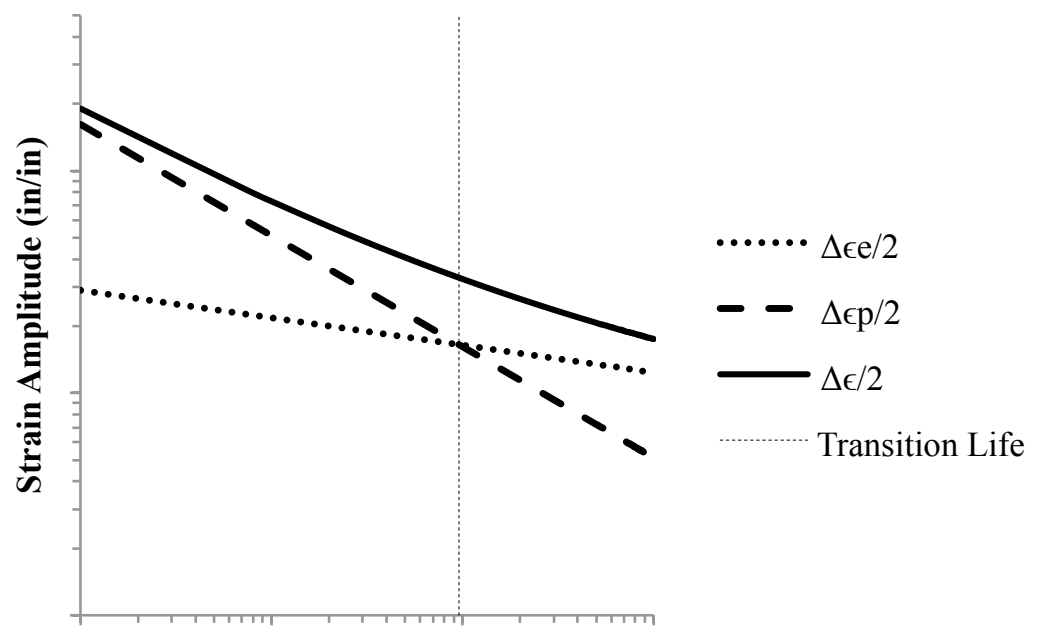

Reversals to Failure, 2Nf (reversals

Figure 1. Typical strain-life curves.

The need to characterize a material's basic properties is also an important lesson. Published values are generally for very specific variations of materials. Depending on manufacturing practices and tolerances, properties such as the elastic modulus, ultimate strength, and yield strength may be very different from the published values.

DME is essentially a second course in strength of materials, currently structured as follows:

- Load and stress analysis (2 weeks)

- Deflection and stiffness (2 weeks)

- Static (stress-based) failure theories (1 week)

- Fatigue (stress-life) (3 weeks)

- 4 case studies (2 weeks, distributed throughout the quarter)

The set of case studies was added to the DME course in the Fall quarter 2011. Each case study involves the design and analysis of a mechanical system. Examples of case studies employed in the course include the design of cable bar bracket, a bearing test rig, and a microphone stand. The Socratic method of study is employed here, where the instructor posed a question, the student provided an answer, followed by another question from the instructor. The case studies are meant to simulate design practices which often occur either informally in the workplace or as a natural process involving cross-disciplinary design teams. 
The exclusion of alternate static and fatigue failure theories from the current curriculum creates a gap in a mechanical engineer's knowledge of failure. With a switch from a quarter based to a semester based academic calendar slated for the Fall of 2013, the extra weeks per term will create the necessary time to teach more than just the high cycle fatigue that is covered now. With a large portion of mechanical engineers at RIT concentrating their coursework in specialized areas such as aerospace and biomedical engineering, the need to describe the behavior of complex alloys that may be subjected to higher stresses becomes even greater. For this work, we have implemented a fatigue lab activity as one of the four case studies in the course.

\section{Laboratory Design}

During the spring of 2012, a laboratory activity involving both LCF and HCF fatigue concepts was created to meet a set of design constraints and allow easy incorporation of this activity into an existing course. To clearly illustrate the impact of low cycle fatigue within the time constraints of a series of lecture periods, rather than a lab, the design constraints included:

- Load capacity of the test system ( $\pm 22,000 \mathrm{lbf})$

- Sample diameter (grips accommodate $\phi 0.39-\phi 0.63$ in)

- Time to run test $(<2$ hours)

- Sample Length (2-6 in)

The material chosen was 1018 steel, made to ASTM standard dimensions with a 0.5 in grip diameter and 0.25 in gage length diameter. Fatigue tests were conducted in load control under fully-reversing axial loading (zero mean stress) on an Instron 8801 servo-hydraulic fatigue test system with $100 \mathrm{~Hz}$ peak frequency capacity. Load amplitude would be calculated depending on whether a test were to be run for high cycle fatigue or low cycle fatigue, and tests were run at 10 $\mathrm{Hz}$, which would provide relatively short tests for low cycle fatigue, and manageable tests for high cycle fatigue. Nine samples were available; three were loaded to fail in the expected highcycle region in tests performed outside of class, with the other six loaded to fail in the low-cycle region with failure expected within the class period.

Tensile tests were performed on the as-received 1018 cold-drawn stock in order to verify the elastic modulus, E, the tensile strength, $\mathrm{S}_{\mathrm{U}}$, and the $0.2 \%$ offset yield strength, $\mathrm{S}_{\mathrm{Y}}$. Table 1 summarizes the tensile test results, including the large variation between actual values for $S_{Y}$ and $\mathrm{S}_{\mathrm{U}}$ and the results available from online material data sources commonly used by students. The experimental values were used for the modeling of the both stress-life fatigue curve and strainlife fatigue curves.

Fatigue test results are shown in Figures 2 and 3. Figure 2 shows that the samples cycled above the transition life correlate well with the high cycle fatigue curve, whereas those that failed at lives less than the transition life deviate from the curve dramatically. In Figure 3, the full strainlife model fits the full range of data better than stress-life alone. This underscores the fact that high cycle fatigue theory is insufficient to describe the behavior of material under similar loading conditions, and therefore that this example can be used with a class to illustrate the importance of understanding the appropriate conditions under which a fatigue model may be applied. 
Table 1. Results from tensile test of fatigue specimen material

\begin{tabular}{|c|c|c|c|}
\hline & Published [8] & Experimental & Difference (\%) \\
\hline $\mathrm{E}(\mathrm{ksi})$ & 29,000 & 28,670 & $1.2 \%$ \\
\hline $\mathrm{S}_{\mathrm{u}}(\mathrm{psi})$ & 70,300 & 92,000 & $24 \%$ \\
\hline $\mathrm{S}_{\mathrm{y}}(\mathrm{psi})$ & 60,200 & 77,000 & $18 \%$ \\
\hline$\% \mathrm{RA}$ & $40 \%$ & $40 \%$ & 0 \\
\hline
\end{tabular}

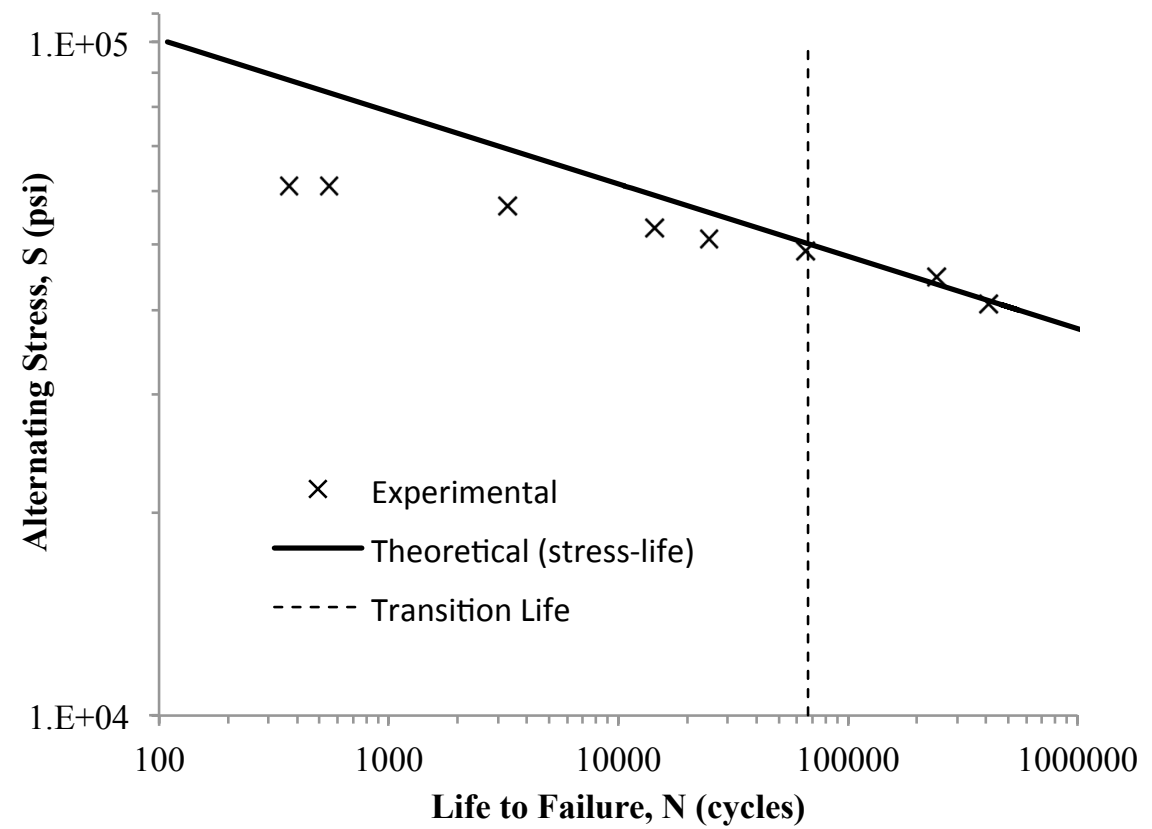

Figure 2. Fatigue Results plotted on stress-life curve.

However, when these data points are plotted against the theoretical strain-life plot, they all correlate quite well (Figure 3). Both the high cycle and low cycle specimens match up well with the total strain curve. Of note is the fact that the 3 highest life data points are those that are dominated by elastic strain, and thus match with both the stress-life and strain-life plots.

Comparing Figures 2 and 3, it is clear to see the very reason why low cycle fatigue theory exists. Overall, better correlation is achieved using strain-life theory rather than stress-life theory. It should be noted that, during testing, buckling of the material was observed, and was most pronounced for the highest-load specimens. This is due to a probable misalignment of the test system. The results of this preliminary testing indicated that this laboratory exercise should help to reinforce the idea that a stress-based approach to fatigue was not always sufficient. 


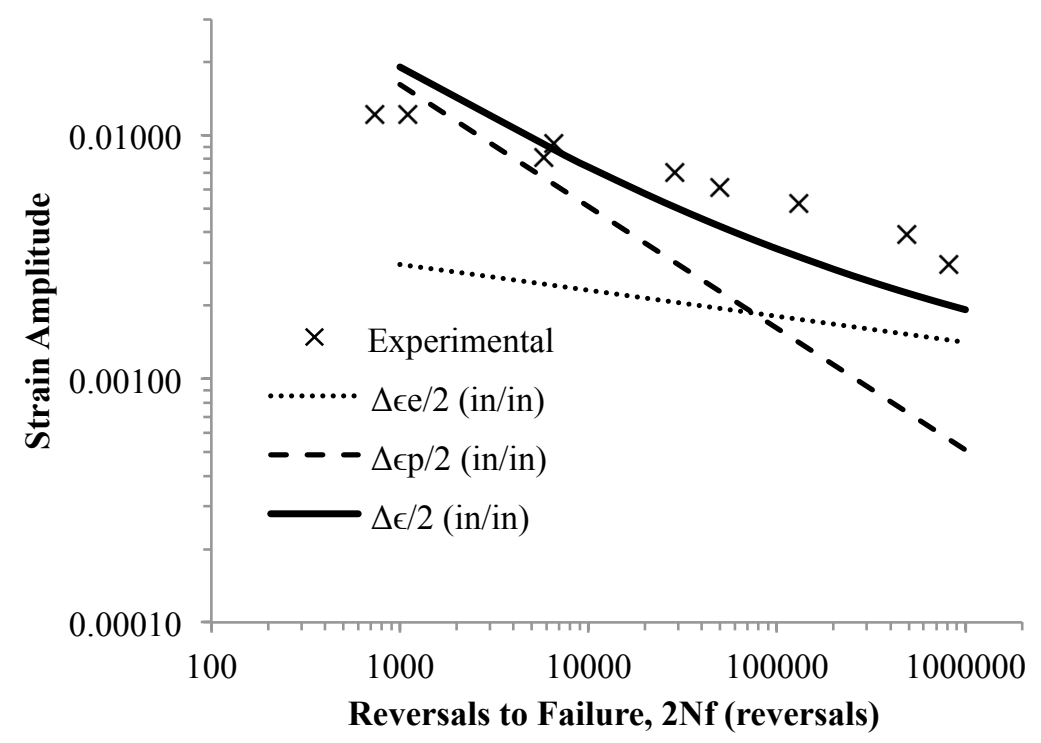

Figure 3. Fatigue results plotted on strain-life curves.

\section{Classroom Implementation}

During Fall 2012, the fatigue lab activity was implemented in class. The material available for the activity was 1065 steel, not 1018 cold-drawn, which presented some challenges: the tensile properties would need to be re-measured and test loads adjusted accordingly. The class was given the tensile data and test specimen geometry, and over the course of two class periods, the students in the class rotated through the lab for fatigue testing. A lab supervisor demonstrated the setup procedure, and the students assisted in running the tests and collecting data.

Table 2 shows the data obtained by the students in the Fall 2012 quarter. Clearly, the tensile data is suspect: steel with a modulus more than twice the typical value is cause for concern.

Additionally, the tensile test results seem to indicate that the as-received material is $50 \%$ stronger than published, yet with nearly the same level of ductility. The fatigue test data, when plotted together with a stress-life fatigue model (Figure 4), seem to indicate that the material is behaving exactly as expected - except that the loads applied should have resulted in low-cycle fatigue behavior, and therefore a poor prediction using stress-life methods. The strain-life model, shown in Figure 5, does a poor job of predicting the experimental data.

As a means of quantitative assessment, Table 3 provides a statistical Student's t-test evaluation of mean class scores on final exam problems pertaining to fatigue life. Each exam problem was worth a maximum of 25 points, and each problem attempted to address a specific concept involving infinite or finite life at a comparable level of difficulty. Essentially identical case studies and identical course lecture material were given to the students in the Fall 2011 and Fall 2012 quarters, and the courses in these successive years were taught by the same instructor. Thus, the only tangible variable introduced to the course is the inclusion of experimental fatigue testing in Fall 2012. For exam problems involving infinite life, the large p-value strongly indicates that the slight drop in class average difference is likely due to chance. For exam problems involving finite life, an unexpectedly statistically significant drop in mean scores was observed in Fall 2012. The finite life exam problem in Fall 2011 involved an S-N curve 
appropriate for steels, while the exam problem in Fall 2012 involved aluminum, which has no endurance limit. Hence, an incomplete understanding of stress-life relations for nonferrous materials may be the explanation for the drop in test scores which likely override the expected benefits of incorporating finite life fatigue testing into the curriculum.

Two qualitative means of assessment for the fatigue test experiments were also done. The first was student responses during the class when the fatigue case study was discussed. Students had difficulty explaining the reason for the ASTM dimensional specification of the test sample, particularly the large radius of curvature between the sample ends and the narrower test region to essentially eliminate stress concentration effects. The students were also willing to accept the erroneous agreement of the test results with stress-based theory, even though it was covered in class that large variability in measured stress-based life occurs for components undergoing shortcycle fatigue. The second means of qualitative assessment was based on feedback from course evaluations. Students were asked to comment on whether the addition of the fatigue test as a course topic was positive or not, and 21 students viewed the experience as positive and 6 viewed it as negative.

Table 2. Fall quarter 2012 test results.

\begin{tabular}{|c|c|c|}
\hline & Published [8] & Experimental \\
\hline E (ksi) & 29,000 & 70,000 \\
\hline $\mathrm{S}_{\mathrm{u}}(\mathrm{psi})$ & 92,100 & 129,000 \\
\hline$\%$ RA & $45 \%$ & $40 \%$ \\
\hline Test \# & Stress Amplitude & Life (cycles) \\
\hline 1 & $62,134 \mathrm{psi}$ & 25,640 \\
\hline 2 & $62,134 \mathrm{psi}$ & 34,060 \\
\hline 3 & $78,126 \mathrm{psi}$ & 11,020 \\
\hline 4 & $78,126 \mathrm{psi}$ & 6,188 \\
\hline 5 & $78,126 \mathrm{psi}$ & 9,740 \\
\hline 6 & $78,126 \mathrm{psi}$ & 6,138 \\
\hline
\end{tabular}

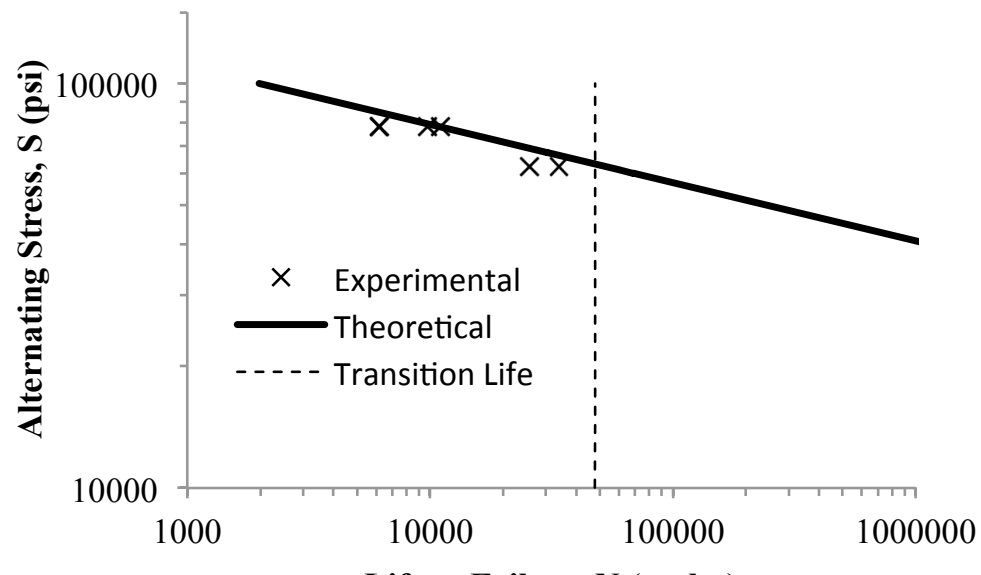

Life to Failure, $N$ (cycles)

Figure 4. Fall quarter 2012 fatigue test results: stress-life. 


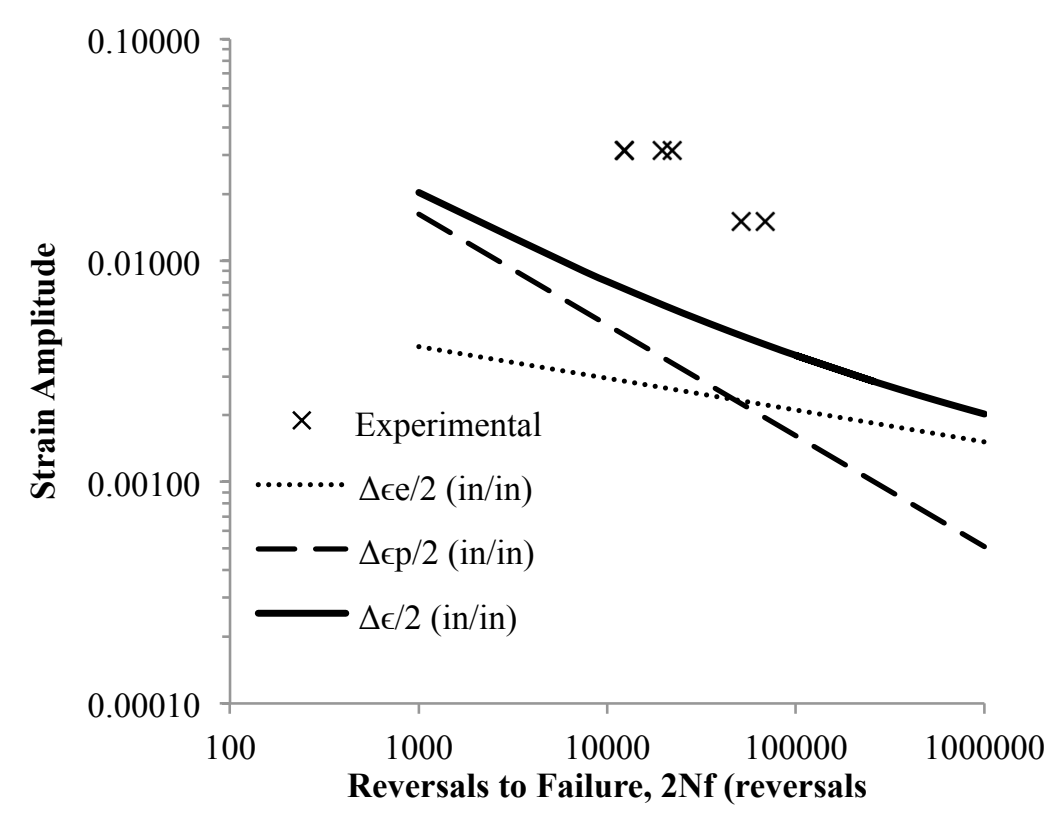

Figure 5. Fall quarter 2012 fatigue test results: strain-life.

Table 3. Statistical Comparison of Mean Exam Problem Scores

\begin{tabular}{|l|c|c|}
\hline Infinite Life problem $(\mathbf{p}=\mathbf{0 . 3 4 8})$ & Fall 2011 & Fall 2012 \\
\hline Number of students & 42 & 34 \\
\hline Mean score (max 25) & 21.79 & 21.21 \\
\hline Standard deviation & 2.97 & 2.23 \\
\hline Finite Life problem (p=0.000002) & Fall 2011 & Fall 2012 \\
\hline Number of students & 42 & 34 \\
\hline Mean score (max 25) & 22.24 & 18.00 \\
\hline Standard deviation & 3.47 & 3.73 \\
\hline
\end{tabular}

\section{Discussion and Future Plans}

In the future, the laboratory and classroom exercises will be repeated with 1018 cold-drawn steel and a non-ferrous material, such as Aluminum. The fact that the tensile data presented to the class was suspect led to opportunity for confusion that, while it raised an interesting point of discussion (validity of available mechanical properties), it distracted from the primary goal of discussion the appropriateness of stress-life vs. strain-life fatigue analysis. With better data, additional statistical assessment of exam problems will be conducted as the course is transformed into the semester curriculum. Additionally, the exam question chosen to measure the success of this activity will include a portion related specifically to the appropriateness of stress-based fatigue modeling in scenarios where strain-based fatigue models may be better suited.

Other possibilities involve the study of ultra-low cycle fatigue and its relation to static failure theory. Material processing effects on fatigue, such as annealing vs. quenching, could also be addressed. 


\section{Summary}

The preliminary specimen designs were successful in yielding results that illustrate the basics of high cycle and low cycle fatigue theory. The stress-based fatigue model was sufficient for high cycle tests, but was insufficient for low cycle tests. While the data gathered during the course implementation made it difficult to make the case for considering other approaches to fatigue life prediction, the authors feel that, with a better choice of material, and more clearly written documentation to avoid poor data collection, this activity is worth pursuing in future offerings of the course.

\section{Acknowledgements}

The authors would like to acknowledge Dave Hathaway for his help in manufacturing the fatigue specimens.

\section{References}

[1] Bannantine, J. A., Comer, J. J., and Handrock, J. L., 1990, Fundamentals of Metal Fatigue Analysis, Prentice Hall, Englewood Cliffs, NY.

[2] Dowling, N.E., 2013, Mechanical Behavior of Materials, Pearson, Upper Saddle River, NJ.

[3] Dues, J., and Le, N., 2006, "High Cycle Fatigue Tester," Proceedings of the 2006 ASEE Annual Conference and Exposition, ASEE.

[4] Sepahpour, B., and Chang, S.-R., 2005, "Low Cycle and Finite Life Fatigue Experiment," Proceedings of the 2005 ASEE Annual Conference and Exposition, ASEE.

[5] Hagigat, C.K., 2005, "Using Commercially Available Finite Element Software for Fatigue Analysis," Proceedings of the 2005 ASEE Annual Conference and Exposition, ASEE.

[6] Elahinia, M., and Ciocanel, C., 2006, "Redeveloping the Mechanics and Vibration Laboratory: A Problem Solving Approach," Proceedings of the 2006 ASEE Annual Conference and Exposition, ASEE.

[7] Ciocanel, C., and Elahinia, M., 2006, “A Problem Solving Approach for Teaching Engineering Laboratories," Proceedings of the 2006 ASME International Mechanical Engineering Congress and Exposition, ASME.

[8] Downloaded from www.matweb.com. 